\title{
Breaking up a pro-survival interaction
}

\section{Cff \\ intravenously \\ administered \\ S63845 \\ exerted dose- \\ dependent \\ anti-tumour \\ activity and \\ induced \\ complete \\ regression in \\ seven out of \\ eight mice}

The pro-survival protein myeloid cell leukaemia 1 (MCL1) is overexpressed in many different types of cancer, but development of small molecule inhibitors for this protein has been challenging. Now, reporting in Nature, Olivier Geneste and colleagues describe a highly specific and potent small molecule inhibitor of MCL1 that has single-agent activity and good tolerability in several cancer models.

MCL1 is a member of the BCL-2 family of proteins, which are key regulators of the mitochondrial apoptotic pathway. They are characterized by the presence of at least one of four BCL-2 homology (BH) domains and can be divided into different functional subgroups. Along with BCL-2, BCL- $\mathrm{X}_{\mathrm{L}}$, BCL-W and BFL, MCL1 belongs to the pro-survival family, whereas the multi-BH-domain-containing effectors $\mathrm{BAX}, \mathrm{BAK}$ and $\mathrm{BOK}$, as well as $\mathrm{BH} 3$-only proteins such as BIM and $\mathrm{BAD}$, have pro-apoptotic functions. In many types of cancer, genetic or epigenetic changes perturb the balance between pro-and antiapoptotic BCL-2 family members to promote survival.

Commitment to apoptosis involves the binding of the $\mathrm{BH} 3$ domain of a pro-apoptotic member to a groove on the surface of the pro-survival proteins. This has inspired the design of $\mathrm{BH} 3$ mimetic drugs, and the BCL-2 inhibitor ABT-199 (venetoclax) received FDA approval for the treatment of chronic lymphocytic leukaemia in 2016.

Using NMR-based fragment screening and structure-guided drug discovery, the authors developed the MCL1 inhibitor S63845. A co-crystal structure showed that it binds to the
BH3-binding groove of MCL1 in a similar site to previously described BH3 mimetic MCL1 inhibitors, but with substantially higher affinity. In vitro experiments using MCL1dependent multiple myeloma (MM) cells showed that 663845 was 1,000fold more effective than a previously described MCL1 inhibitor, and co-immunoprecipitation, together with CRISPR/Cas9 knockout experiments, showed that it kills cells through activation of the BAX- or BAK-dependent mitochondrial pathway of apoptosis.

The authors showed that a substantial proportion of cell lines derived from haematological cancers, as well as freshly derived samples from patients with acute myeloid leukaemia (AML), are exquisitely sensitive to S63845. In mice with human MM xenografts, intravenously administered S63845 exerted dose-dependent anti-tumour activity and induced complete regression in seven out of eight mice. Even at the highest efficacious dose tested, the drug was well tolerated. Similar results were observed in an AML xenograft model and in $\mathrm{E} \mu$-Myc transgenic mice, in which both tumour and normal cells express mouse MCL1 protein and thus have the same affinity for S63845.

The drug was also tested in cell lines derived from solid tumours; a third of melanoma and breast cancer cell lines were sensitive to S63845, and sensitivity was inversely correlated with BCL- $\mathrm{X}_{\mathrm{L}}$ mRNA expression. Consistent with previous reports that inhibitors of oncogenic kinases can sensitize tumour cells to BH3-mimetic drugs targeting BCL-2 and/or BCL- $\mathrm{X}_{\mathrm{L}}$, co-treatment with inhibitors of HER2 (also known as

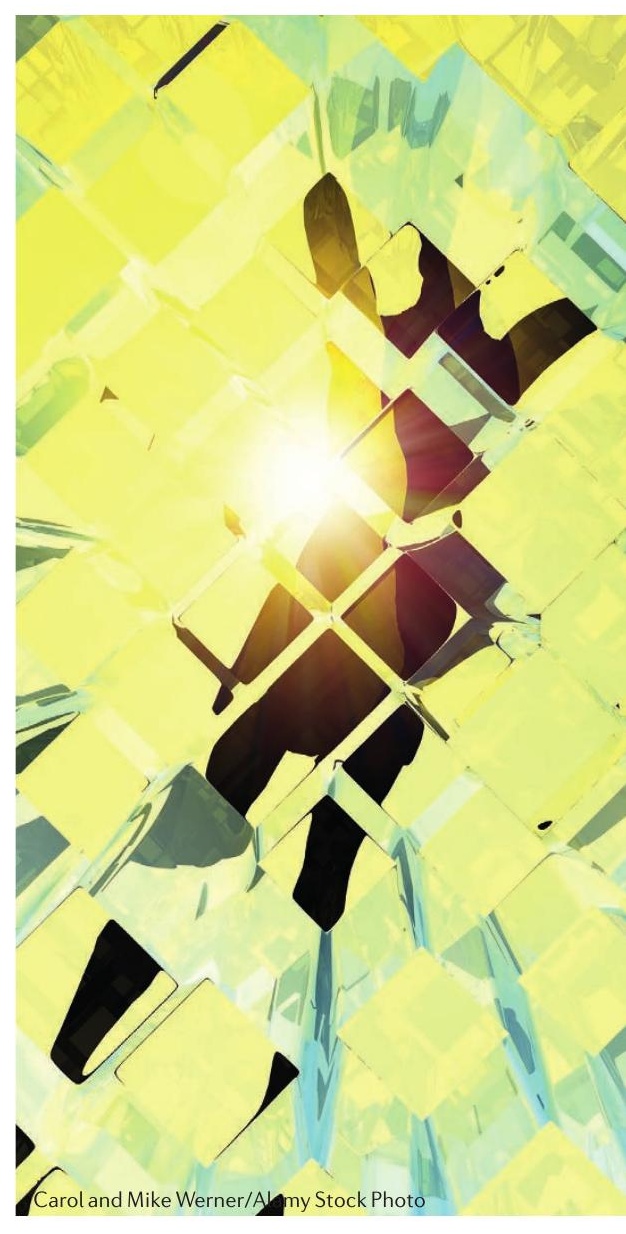

ERBB2), epidermal growth factor receptor (EGFR), MEK or RAF induced potent cytotoxic responses.

These results demonstrate that MCL1 is a druggable target and that direct inhibition of MCL1 can be tolerated and is efficacious in a broad range of tumours.

Alexandra Flemming

This article also appears in Nat. Rev. Cancer

ORIGINAL ARTICLE Kotschy, A. et al.

The MCL1 inhibitor $\mathbf{5 6 3 8 4 5}$ is tolerable and

effective in diverse cancer models. Nature $\mathbf{5 3 8}$, 477-482 (2016) 